\title{
SOME RESIDUALLY FINITE GROUPS SATISFYING LAWS
}

\author{
YVES DE CORNULIER AND AVINOAM MANN
}

Abstract. We give an example of a residually- $p$ finitely generated group, that satisfies a non-trivial group law, but is not virtually solvable.

Denote by $F_{n}$ the free group on $n$ generators. Recall that, given a group word $m\left(x_{1}, \ldots, x_{n}\right) \in F_{n}$, a group $G$ satisfies the law $m=1$ if for every $u_{1}, \ldots, u_{n} \in G$, $m\left(u_{1}, \ldots, u_{n}\right)=1$. Given a set $\mathscr{S}$ of group laws, the $n$-generator free group in the variety generated by $\mathscr{S}$ is the quotient of $F_{n}$ by the intersection of all kernels of morphisms of $F_{n}$ to a group satisfying all the group laws in $\mathscr{S}$. Taking the quotient by the intersection of all finite index subgroup (resp. of $p$-power index), we obtain the restricted (resp. $p$-restricted) $n$-generator free group in the variety generated by $\mathscr{S}$.

The celebrated Tits Alternative states that if $G$ is a finitely generated linear group over any field, then either $G$ contains a non-abelian free subgroup, or it is virtually solvable (i.e. contains a solvable subgroup of finite index). It follows that if such a group $G$ satisfies a nontrivial group law, it is virtually solvable. It is natural to ask to what extent the assumption of linearity can be relaxed. Can we, for instance, replace linearity by residual finiteness? Here we show that this is not possible, even under the assumption that $G$ is residually- $p$ (i.e. residually a finite $p$-group). We provide several constructions. The results we obtain are probably known to the specialists; however, to the best of our knowledge, they do not seem to appear in the literature.

For any $q \in \mathbf{N}$, let $G_{q}$ be the restricted free 2-generator group in the variety generated by the group law $[x, y]^{q}=1$.

We begin by the following elementary result:

Theorem 1. For $q=30, G_{q}$ is a 2-generator, residually finite group that satisfies a nontrivial group law, but is not virtually solvable.

Proof. The only nontrivial verification is that $G_{30}$ is not virtually solvable. To show this, it suffices to show that, for every $n, G_{30}$ has a finite quotient having no solvable subgroup of index $\leq n$.

Start with $I=\mathrm{Alt}_{5}$. Then $|I|=60$ and any solvable subgroup of $I$ has order $\leq 12$. Therefore, for every $m$, any solvable subgroup of $I^{m}$ has order $\leq 12^{m}$, therefore index $\geq 5^{m}$.

Now, for all $k \geq 2$, the wreath product $I 乙 C_{k}=I^{k} \rtimes C_{k}$ is generated by 2 elements: the element $(1, z)$, where $z$ is a generator of $C_{k}$ (we now identify $z$ and $(1, z)$ ), and the element $\sigma=((s, t, 1, \ldots, 1), 1)$, where $s$ has order $2, t$ has order 3 , and $s$ and $t$ generate $I$. Indeed, $\sigma^{3}=(s, 1, \ldots, 1), z^{-1} \sigma^{4} z=(t, 1 \ldots, 1)$, so that $\sigma$ and $z$ generate $I$ I $C_{k}$. Now $I$ \& $C_{k}$ has derived subgroup of exponent 30 , hence it is a quotient of $G_{30}$.

Date: April 24, 2006. 
Remark 2. The wreath product $\operatorname{Alt}_{5}<\mathbf{Z}$ is not residually finite; actually it has no residually finite quotient bigger than $\mathbf{Z}$ [Gr57].

By taking the profinite completion, we obtain:

Corollary 3. There exists a profinite group, topologically finitely generated, that satisfies a non-trivial group law, and is not virtually prosolvable.

Theorem 1 can be strengthened by demanding the group to be residually- $p$. For this purpose, we need to appeal to a deep result of Y.P. Razmyslov (see [VL93]).

Theorem 4 (Razmyslov). For every prime power $q \geq 4$, there exist finite groups of exponent $q$ and arbitrarily large solvability length.

Given $n \in \mathbf{N}$ and a set $X$, denote by $B(X, n)$ the free group of exponent $n$ on the generators $\left(u_{x}\right)_{x \in X}$. Let $R(X, n)$ be the restricted free group of exponent $n$ on the generators $\left(u_{x}\right)_{x \in X}$; namely, the quotient of $B(X, n)$ by the intersection of all its finite index subgroups.

Remark 5. There is a canonical isomorphism between $R(X, n)$ and the direct limit $\lim _{F} R(F, n)$, where $F$ ranges over all finite subsets of $X$.

Indeed, for finite $F \subset X$, there is a natural split morphism $B(F, n) \rightarrow B(X, n)$, inducing a split (in particular, injective) morphism $i_{F}: R(F, n) \rightarrow R(X, n)$. This induces a morphism of the direct $\operatorname{limit}_{\longrightarrow} \lim _{F} R(F, n) \rightarrow R(X, n)$. As a direct limit of injective morphisms, it is injective; it is trivially surjective since all marked generators are in the image.

In particular, for all $n$ such that groups of exponent $n$ are locally finite (this is known for $n \leq 4$ and $n=6), R(X, n)=B(X, n)$.

If $G$ is any group, then $G$ acts on $R(G, n)$ by shifting the generators.

Proposition 6. Suppose that $G$ is residually finite (resp. residually-p). Then so is $G \ltimes R(G, n)$ for all $n \in \mathbf{N}$ (resp. for every $n=p^{k}$ for some $k \in \mathbf{N}$ ).

Proof. Let $(g, x)$ belong to $G \ltimes R(G, n)$ let us show that there exists a residually finite quotient of $G$ in which $(g, x)$ has a nontrivial image. If $g \neq 1, G$ is such a quotient. Suppose that $g=1$. Writing $x$ as a word in the generators $\left(u_{g}\right), g \in G$, involves only a finite subset $B$ of $G$. By residual finiteness of $G$, there exists a finite quotient $G / N$ of $G$ such that the quotient morphism is injective in restriction to $B$. It extends to a morphism of $G \ltimes R(G, n)$ onto $G / N \ltimes R(G / N, n)$, whose restriction to $R(B, n)$ is injective (this follows from Remark 5 ). It follows that the image of $x$ in $G / N \ltimes R(G / N, n)$ is nontrivial. Finally, $G / N \ltimes R(G / N, n)$ is residually finite ${ }^{1}$ since it contains $R(G / N, n)$ as a subgroup of finite index.

The proof of the statement for residually- $p$ groups is similar.

Remark 7. A similar result holds if we replace the restricted free groups of exponent $n$ by the restricted (or $p$-restricted) free groups on any variety.

Theorem 8. For all $n, \mathbf{Z} \ltimes R(\mathbf{Z}, n)$ is 2-generated, residually finite, residually-p if $n$ is a power of $p$, and satisfies the group law $[x, y]^{n}=1$. If $n=4,5$ or $n \geq 7$, it is not virtually solvable.

\footnotetext{
${ }^{1} \mathrm{By}$ the solution to the restricted Burnside problem, it is even finite. But we do not need this deep result due to Zelmanov (see [VL93]), and our argument is preferable in view of Remark 7.
} 
Proof. It is clearly generated by $(1,1)$ and $\left(0, u_{0}\right)$. The statement on residual finiteness follows from Proposition 6 . The group law $[x, y]^{n}=1$ is trivially satisfied since $\mathbf{Z}$ is abelian. The last statement follows from Theorem 4 , which is equivalent to the following statement: for every prime-power $q \geq 4, R(\mathbf{Z}, q)$ is not virtually solvable. Indeed, if it were virtually solvable, there would be a bound on the length of solvability of finite groups of exponent $q$. It immediately generalizes to any non-necessarily prime-power $q \geq 7$ : indeed, such a number has a divisor that is either 4, 9, or a prime $\geq 5$.

Remark 9. In the remaining cases, namely when $n \leq 3$ or $n=6$, the free Burnside group $B(\mathbf{Z}, n)$ is solvable and locally finite; for $n \leq 2$ it is clearly abelian; for $n=3$ it is metabelian and for $n=6$ it is 5 -solvable (more precisely, it is (exponent 3)-by(exponent 2)-by-(exponent 3)), by a result of M. Hall [MH58].

As an application, by taking the pro- $p$ completion, we answer a question asked by E. Breuillard and T. Gelander in an early version of [BG04].

Corollary 10. There exists a pro-p-group, topologically finitely generated, that satisfies a non-trivial group law, and is not virtually solvable.

Let us provide a second construction.

Let $F$ be a free group of rank 2 , and fix a prime power $q=p^{a} \geq 4$. By Theorem 4 , for every $n$, there exists $k$ and a finite $k$-generated group of exponent $q$ that is not $n$-solvable. Choose a normal subgroup $N_{n}$ of $F$ such that $F / N_{n}$ is abelian of order $r$, where $r \geq k-1$ is a power of $p$. Let $K_{n}$ be the smallest normal subgroup of $N_{n}$ with a finite factor group of exponent $q$; note that $K_{n}$ is characteristic in $N_{n}$ and is therefore normal in $F$. Besides, since $N$ is free of rank $r+1$, by the assumption on $k, N_{n} / K_{n}$ is not $n$-solvable. Now setting $G=F / \cap K_{n}, G$ is 2 -generated and residually- $p$; its derived subgroup has exponent $q$ but is not virtually solvable. Note that this provides another proof that the group $G_{q}$ introduced above is not virtually solvable for all prime power $q \geq 4$ (and therefore for every integer $q \geq 4, q \neq 6$ ).

We now give a third construction, of independent interest, relying on the following theorem from [NN59]; we do not quote it in the utmost generality.

Theorem 11 (B.H. Neumann and H. Neumann, 1959). Let $G$ be a countable group. Then there exist cyclic groups $B, C$, and an embedding $i$ of $G$ into the unrestricted wreath product $Q=(G) C) 2 B$ so that $i(G)$ is contained in the second derived subgroup $\Gamma^{\prime \prime}$ of a two-generator subgroup $\Gamma$ of $Q$ (in particular, every group law satisfied by $G$ is satisfied by $\left.\Gamma^{\prime \prime}\right)$.

Moreover,

(1) if $G$ is finitely generated, we can choose for $B$ any cyclic group of sufficiently large order $k \in \mathbf{N} \cup\{\infty\}$ (say ${ }^{2}, k \geq 4 m-1$, if $G$ is generated by $m$ elements), and

(2) if $G$ is generated by elements whose orders divide $n$, we can choose $C=\mathbf{Z} / n \mathbf{Z}$ (in particular, we always can choose $C=\mathbf{Z}$ ).

Here is our third construction.

\footnotetext{
${ }^{2}$ Indeed, if $k \geq 4 m-1$, then the elements $b_{1}=1, b_{2}=3, \ldots b_{m}=2 m-1$ of $\mathbf{Z} / k \mathbf{Z}$ satisfy (4.3) of [NN59].
} 
Let $H_{q}$ be the free 2-generator group in the variety generated by the group law $[[x, y],[z, t]]^{q}=1$. Let $H:=\left(H_{q}\right)_{r p}$ be the quotient of $G$ by the intersection of all its normal subgroups of $p$-power index; it is trivially residually- $p$. We claim that, for $q \geq 4$, a power of $p$, it is not virtually solvable.

For every $n$, by Theorem 4, there exists a finite group $F$ of exponent $q$, and of solvability length $\geq n$. By Theorem $11, F$ embeds in the second derived subgroup of a 2-generator subgroup $P$ of $\left(F \nmid C_{p^{k}}\right) \backslash C_{p^{k}}$ for sufficiently large $k$. Then $P$ is a $p$-group and it is a quotient of $H_{q}$, therefore also of $H$. Suppose that $H$ has a normal solvable subgroup of finite index $r$ and solvability length $h$. Then $P$ has a normal subgroup $Q$ of index at most $r$ and solvability length at most $h$. Then $P$ itself has solvability length at most $h+r$, which is impossible if $n$ is large enough.

Question 12. Does there exist a residually finite, finitely presented group, that satisfies a nontrivial group law but is not virtually solvable?

Remarks 13. - This question is not trivial at all even without the residual finiteness assumption. A.Olshanskii and M.Sapir [OS02] have constructed, for large $n$, a finitely presented, non virtually solvable group (actually non-amenable), whose derived subgroup has exponent $n$. By the solution to the restricted Burnside problem, their group is not residually finite, since it contains an infinite, finitely generated, finite exponent group.

- If we also drop the finite presentation assumption, there are many examples. One of the simplest (but not the best known) is given by the standard wreath product $F \nmid \mathbf{Z}$, where $F$ is any finite, non-solvable group.

- Again using the solution to the restricted Burnside problem, the residually finite groups whose derived subgroup has finite exponent are (locally finite)-by-abelian.

If $q$ is a prime power, then the free restricted group in the variety generated by the group law $[x, y]^{q}$ is also (locally finite)-by-abelian, by [Sh99] (a related result appears in [Sh02]). In particular, all these groups are elementary amenable. This motivates the following question:

Question 14. Does there exist a finitely generated, residually finite group, that is not (elementary) amenable and satisfies a nontrivial group law? What about the free restricted group in the variety generated by, for suitable $n$, the group law $[x, y]^{n}$ ? $[[x, y],[z, t]]^{n} ?$

We can ask a similar question concerning semigroup laws.

Question 15. Does there exist a topologically finitely generated profinite group (resp. pro-p-group) that does not contain any free semigroup, but is not virtually nilpotent?

Remark 16. A deep result of J.Semple and A.Shalev [SS93] states that a residually finite, finitely generated group satisfying a nontrivial semigroup law is virtually nilpotent.

Remark 17. The first Grigorchuk group is a finitely generated, residually-2 torsion group, hence does not contain any free semigroup, and is not virtually nilpotent. However, its pro-2 completion contains nonabelian free subgroups.

Actually, The Grigorchuk group does not satisfy any non-trivial group law. This raises our final 
Question 18. Can a finitely generated group satisfying a non-trivial law have intermediate growth?

\section{REFERENCES}

[BG04] E. Breulllard, T. Gelander. A topological Tits alternative, Preprint 2004, to appear in Ann. of Math.

[Gr57] K. W. Gruenberg. Residual properties of infinite soluble groups, Proc. London Math. Soc. (3) 7, 1957, 29-62.

[MH58] M. HALL. Solution of the Burnside problem for exponent six. Illinois J. Math. 2 (1958), 764-786.

[Nn59] B. H. Neumann, H. Neumann. Embedding Theorems for Groups, J. London Math. Soc. 34 (1959), 465-479.

[OS02] A. OlShANSKII, M. SAPIR. Non-amenable finitely presented torsion-by-cyclic groups. Publ. Math. Inst. Hautes Études Sci. 96 (2002), 43-169.

[Sh99] P. Shumyatsky. On groups with commutators of bounded order, Proc. Amer. Math. Soc. 127 (1999), 2583-2586.

[Sh02] P. ShumYatsky. Commutators in residually finite groups, Monatsh. Math. 137 (2002), 157-165.

[SS93] J. Semple, A. Shalev. Combinatorial Conditions in Residually Finite Groups, I, J. Algebra 157, 1 (1993), 43-50.

[Vl93] M.R. Vaughan-Lee. The restricted Burnside Problem, 2nd ed., Oxford Univ. Press, 1993.

Y.C. École Polytechnique Fédérale de Lausanne (EPFL), Institut de Géométrie, Algèbre et Topologie (IGAT), CH-1015 Lausanne, Switzerland

E-mail address: yves.cornulier@ens.fr

A.M. Einstein Institute of Mathematics, Hebrew University, Jerusalem 91904, ISRAEL

E-mail address: mann@math.huji.ac.il 